\title{
Functioning of unique nitrile-detoxifying system in soil xenobiotic degrader Rhodococcus rhodochrous: a whole-genome transcriptomic approach
}

\author{
Konstantin V Lavrov \\ NRC "Kurchatov institute - \\ GosNIIgenetika" \\ lavrov.ko@gmail.com \\ Andrey D Novikov \\ NRC "Kurchatov institute - \\ GosNIIgenetika" \\ alexm19@mail.ru
}

\author{
Artem S Kasianov \\ Vavilov Institute of General Genetics, \\ Moscow, Russia \\ artem.kasianov@gmail.com
}

\author{
Alexander S Yanenko \\ NRC "Kurchatov institute - \\ GosNIIgenetika" \\ yanenko@genetika.ru
}

\author{
Tatyana I Kalinina \\ NRC "Kurchatov institute - \\ GosNIIgenetika" \\ tatyana.i.kalinina@googlemail.com
}

\begin{abstract}
Organic nitriles are significant participants of interactions between soil living organisms, thus their synthesis and degradation are of interest. Using transcriptomic approach, we revealed a genetic cascade, realizing nitrile degradation in powerful nitrile degrader - Rhodococcus rhodochrous. We also hypothesized a genetic "network" for nitrile/amide utilization, connected with cobalt homeostasis.
\end{abstract}

Keywords - Rhodococcus, nitrile, amidase, cobalt, transcriptome, pathway regulation

\section{Motivation and aim}

Organic nitriles, both natural (mainly plant-derived [1]) and artificial (pesticides), are widely presented in soil. Nitriles are toxic, thus their detoxifying is an important part of interactions within soil micro- and macrobiota. Nitrilemediated interactions can be complex, including pest protection or variants of cooperation. An examples of possible complexity are that plant growth hormone indoleacetic acid can be synthesized as a result of enzymatic hydration of indole acetonitrile in bacteria, or, nitriles can be synthesized by bacteria via aldoxime dehydratase activity

Bacteria, which considered being main degraders of nitriles, are able to do it using two catabolic pathways starting with a nitrile hydratase/amidase system (NH/AM) or with nitrilase [3, 4]. Known xenobiotic degraders Rhodococcus bacteria - are also known hosts for both pathways. Remarkable features of cobalt-dependent NH/AM system in Rhodococcus are fast, nitrile-dependent switching on, very high synthesis of NH/AM enzymes (up to $50 \%$ of soluble intracellular proteins), and very high enzyme activity of $\mathrm{NH}$ [5]. Due to this unique combination of features, Rhodococcus species possessing cobalt-dependent NH/AM system are interesting representatives of soil nitriledegrading community.

A few examples of such system are known up to date, and its genetic regulation is poorly studied. It was only reported that cobalt ions and amides (products of $\mathrm{NH}$ activity) can activate transcription of $\mathrm{NH} / \mathrm{AM}$, and roles of three regulator genes located near NH were revealed [6]. We aim to uncover a more wide genetic "network" which participates in regulation and functioning of NH/AM system in Rhodococcus, and here we report a whole-genome transcriptomic approach to solve this task.

\section{Methods}

R. rhodochrous strain $\mathrm{M} 8$, containing $\mathrm{NH}$ and $\mathrm{AM}$ genes [7], was grown on four variants of glucose containing minimal medium, with following additions: (1) $\mathrm{NH}_{4} \mathrm{Cl}$, (2) $\mathrm{NH}_{4} \mathrm{Cl}+$ $\mathrm{CoCl}_{2}$, (3) urea, (4) urea $+\mathrm{CoCl}_{2}$. The first was "blank" variant without activators of NH/AM, and in other cobalt/urea were probable independent (in 2 and 3 ) or cooperative (in 4) activators. Cells were collected at both mid-exponential and stationary phases of growth. Thus, 8 variants of cells were obtained, each as a sum of 10 independent reproducible growth experiments.

Total RNAs were extracted, quality controlled, separated on two aliquots, and resulting 16 samples were further processed independently. After clean-up from ribosomal RNA, cDNA libraries were prepared, and further sequenced on Illumina HiSeq 2500 platform (at Genotek, Moscow, Russia). Reads were mapped on $R$. rhodochrous M8 genome, and relative transcription levels (RTL) of genes were calculated with DESeq2 software. RTLs were expressed in RPKM units, and reflects, in principle, the following ratio: (number of reads mapped on ORF) / (number of all reads in the sample * length of ORF). Differences in RTLs between growth conditions were calculated as fold change $\left(\mathrm{RTL}_{1} / \mathrm{RTL}_{2}\right)$.

\section{Results general observations}

In all growth conditions, minimal RTLs within the genome were approx. 10, and maximal - 16000 - 100000 units (max. 10000 -fold difference). RTLs of approx. $70 \%$ of genes were below 100, and of approx. 5\% - higher than 1000 units. Between growth conditions, fold change of RTLs of $90-99 \%$ genes were lower than 10, and maximal fold change was 250 .

Growth conditions $1\left(\mathrm{NH}_{4}{ }^{+}\right)$resulted in lowest RTLs of majority of genes, and in 2-4 conditions (with NH/AM activators) RTLs of many genes increased up to 250-fold. RTLs of very few genes decreased in 2-4, no more than $10-$ fold (except cluster of 5 ribosomal proteins genes, which decreased 40-fold). Significant differences in RTLs between 2-4 and 1 variant were observed only at exponential growth phase (log-phase). Data from log-phase was described below, if other not indicated. Variant 1 of growth conditions was chosen as blank not only for NH/AM RTLs comparison, but for comparison of RTLs throughout the genome. 
Response of $\mathrm{NH}$ and AM to activators

Of two NHs presented in M8 genome, Co-type and Fetype, only first transcribed at significant levels $(500-100000)$ and varied between growth conditions. RTLs of the last were below 80 and practically constant. Cobalt itself did not activate $\mathrm{Co}-\mathrm{NH}$, and urea itself activated it by 40 fold. Co + urea conditions were the best for $\mathrm{NH}$ activation in log-phase (125-fold). In these conditions at stationary phase, RTL of Co$\mathrm{NH}$ remained high (1,3-fold change comparing to log-phase). Also, RTLs of NH were greatly higher than RTLs of all other genes (approx. 90 000, 10-fold higher than highest other genes (11 000 for cold-shock protein)).

Of 15 available AMs, two AMs (formamidase $f m d 5$ and aliphatic amidase amiE) activated too in these conditions (11 and 25-fold). RTLs of fmd5 and amiE further increased at stationary phase, comparing to log-phase (5- and 1.5-fold change). We suggest that these AMs are involved in biochemical cascade with $\mathrm{Co}-\mathrm{NH}$. In it, an amide starts to appear at log-phase as a result of NH activity, and then AMs continued to degrade it to acid.

RTLs of these AMs were relatively high (11 000 and 3700 ), thus felling into $0.4 \%$ portion of highly transcribing genes. A portion of NH/AM transcripts in total mRNA was as high as $23 \%$, indicating a high significance of this pathway to bacterium. Interestingly, both AMs were much more induced by urea, than by urea + cobalt (100300-fold versus 11-25-fold, correspondingly). This indicates that the AMs can be involved in other yet unknown biochemical cascade (s). Taking in account this observation, and also a presence of $\mathrm{Fe}-\mathrm{NH}$ and several other AMs, which are silent in given conditions, we can suggest a multiple, intersecting and branched nitrile detoxifying pathway in Rhodococcus bacteria.

\section{Response of other genes to activators}

Cobalt itself activates to some extent many genes throughout the genome (5-15-fold change). A few of it seemed to be related to cobalt (uptake, efflux, chelation, and biosynthesis of cobalamin), but majority are not, including clusters of probable prophages. When urea is present too, the majority of these not related to cobalt genes are again switched off. We suggest that cobalt causes massive stress response, which is significantly decreased when $\mathrm{NH}$ is synthesized and cobalt is incorporated into its active center.
Urea itself activates much smaller number of genes, among which are mainly probable genes of urea/ammonia transport and utilization, and, surprisingly, a cobalt-related genes - cobalamin biosynthesis cluster of 4 genes, including cobalt transporters (100-fold change), and separate cobalamin biosynthesis genes cbiX, cobW, cbiM (10-fold change). This observation can indicate possible existence of unknown regulation of cobalamin biosynthesis, and, also possible interconnection between pathways of cobalt and urea in the cell.

\section{Acknowledgment}

This work was financially supported by the Ministry of Education and Science of Russian Federation (Contract No. 075-15-2019-1658, creation and development of the "Kurchatov Genomic Center").

\section{References}

[1] Fraser F. Fleming. Nitrile-containing natural products. Natural Product Reports, 1999,16, 597-606, https://doi.org/10.1039/A804370A

[2] Ken-Ichi Oinuma, Yoshiteru Hashimoto, Kazunobu Konishi, Masahiko Goda, Takumi Noguchi, Hiroki Higashibata, Michihiko

[3] Kobayashi. Novel Aldoxime Dehydratase Involved in CarbonNitrogen Triple Bond Synthesis of Pseudomonas chlororaphis B23. J Biol Chem, 278(32), 29600-8, 2003 Aug 8, DOI: 10.1074/jbc.M211832200

[4] Yanenko, Alexander, Osswald, Steffen. Hydrolysis of Nitriles to Amides. in Enzyme Catalysis in Organic Synthesis, 2012. pp. 531-544 https://doi.org/10.1002/9783527639861.ch13

[5] Oßwald, Steffen, Yanenko, Alexander. Hydrolysis of Nitriles to Carboxylic Acids. in Enzyme Catalysis in Organic Synthesis, 2012. pp 545-559, https://doi.org/10.1002/9783527639861.ch14

[6] Konstantin V Lavrov, Anna O Shemyakina, Elena G Grechishnikova, Andrey D Novikov, Tatyana I Kalinina and Alexander S Yanenko. In vivo metal selectivity of metal-dependent biosynthesis of Co-type nitrile hydratase in Rhodococcus bacteria: a new look at the nitrile hydratase maturation mechanism? Metallomics, 2019, 11, 1162. https://doi.org/10.1039/C8MT00129D

[7] K. V. Lavrov, A. O. Shemyakina, E. G. Grechishnikova, A. D. Novikov, D. D. Derbikov, T. I. Kalinina, A. S. Yanenko. New cblA gene participates in regulation of cobalt-dependent transcription of nitrile hydratase genes in Rhodococcus rhodochrous. Research in Microbiology, 2018, May Jun;169(4-5):227-236, https://doi.org/10.1016/j.resmic.2018.03.006

[8] Novikov AD, Lavrov KV, Kasianov AS, Gerasimova TV, Yanenko AS. Draft genome sequence of Rhodococcus sp. Strain M8, which can degrade a broad range of nitriles. Microbiology Resource Announcements 2018;6. 